\title{
Assessment of knowledge, practice and attitude towards prostate cancer screening among male patients aged 40 years and above at Kitwe Teaching Hospital, Zambia
}

\author{
Sakala Gift ${ }^{1^{*}} \mathbb{0}$, Kasongo Nancy ${ }^{2}$ and Mwanakasale Victor ${ }^{1}$
}

\begin{abstract}
Background: Prostate cancer is a leading cause of cancer death in men. Evaluating knowledge, practice and attitudes towards the condition is important to identify key areas where interventions can be instituted.

Methods: This was a hospital-based descriptive cross-sectional study aimed at assessing knowledge, practice and attitude towards prostate cancer screening among male patients aged 40 years and above at Kitwe Teaching Hospital, Zambia.

Results: A total of 200 men took part in the study (response rate $=100 \%)$. Of the 200 respondents, $67(33.5 \%$ ) had heard about prostate cancer and 58 (29\%) expressed knowledge of prostate cancer out of which $37(63.8 \%)$ had low knowledge. Twenty-six participants (13\%) were screened for prostate cancer in the last 2 years. $98.5 \%$ of the participants had a positive attitude towards prostate cancer screening. Binary logistic regression results showed that advanced age $(p=0.017)$, having secondary or tertiary education $(p=0.041)$, increased knowledge $(p=0.023)$ and family history of cancer $(p=0.003)$ increased prostate cancer screening practice. After multivariate analysis, participants with increased knowledge $(p=0.001)$ and family history of cancer $(p=0.002)$ were more likely to practice prostate cancer screening.
\end{abstract}

Conclusion: The study revealed low knowledge of prostate cancer, low prostate cancer screening practice and positive attitude of men towards prostate cancer screening. These findings indicate a need for increased public sensitization campaigns on prostate cancer and its screening tests to improve public understanding about the disease with the aim of early detection.

Keywords: Knowledge, Practice, Attitude, Prostate cancer screening, Digital rectal examination, Prostate-specific antigen

\section{Background}

Prostate cancer, or adenocarcinoma of the prostate as it is called in some settings, can be described as cancer of the prostate gland.

\footnotetext{
*Correspondence: giftsakala74@gmail.com

${ }^{1}$ Michael Chilufya Sata School of Medicine, The Copperbelt University,

P.O. Box 71191, Ndola, Zambia

Full list of author information is available at the end of the article
}

The prostate is a small fibromuscular accessory gland of male reproductive system weighing about $20 \mathrm{~g}$. It is located posterior to the pubic symphysis, superior to the perineal membrane, inferior to the bladder and anterior to the rectum. It produces and secretes proteolytic enzymes into semen, to facilitate fertilization $[1,2]$.

Prostate cancer is characterized by both physical and psychological symptoms [3]. Early-stage prostate cancer is usually asymptomatic [4]. More advanced disease has similar symptoms with benign prostate conditions such 
as weak or interrupted urine flow, hesitancy, frequency, nocturia, hematuria or dysuria. Late-stage prostate cancer commonly spreads to bones and cause pain in the hips, spine or ribs [4]. The 2 commonly used screening methods for prostate cancer are digital rectal examination (DRE) and prostate-specific antigen (PSA) test.

Prostate cancer is one of the leading causes of cancerrelated deaths among males globally [4]. The 2018 Global Cancer Project (GLOBOCAN) report estimated 1276 106 new cases in 2018, representing 7.1\% of all cancers worldwide [5]. The report further estimated the number of deaths due to prostate cancer at 358989 , representing $3.8 \%$ of all cancers globally. It was thus ranked the second most common cancer and the fifth leading cause of cancer death in men. The American Cancer Society 2019 report showed that an estimated 174,650 new cases of prostate cancer would be diagnosed in the USA during 2019 [4]. The report further stated that an estimated 31,620 deaths from prostate cancer would occur in 2019. It further put the incidence of prostate cancer to about $60 \%$ higher in blacks than in whites suggesting a genetic predilection to the cancer.

Africa is no exception to this global trend of high incidence and mortality of prostate cancer with age-standardized incidence and mortality rates of 26.6 and 14.6 per 100000 men, respectively [5]. This placed prostate cancer as the third most common cancer among both sexes and the fourth leading cause of all cancer deaths among both sexes in the region. Current statistics on Zambia indicate that Zambia has one of the world's highest estimated mortality rates from prostate cancer $[6,7]$. The age standardized incidence and mortality rates from prostate cancer are at 45.6 and 28.4 per 100,000 men, respectively [5].

Although the causes of prostate cancer are not yet fully understood, it is thought that advanced age (above 50 years), positive family history of prostate cancer and an African-American ethnic background are risk factors $[4,8]$.

In mitigating the effects of diseases like prostate cancer, evaluating knowledge, practice and attitudes towards the condition is important to identify key areas where interventions can be instituted. For instance, studies in other countries that accessed these factors were able to identify the role that health workers and political will could play in increasing knowledge and screening for prostate cancer [8-10]. Furthermore, low level of awareness about prostate cancer or the complete lack of it has been identified as the cause of late presentation and poor prognosis $[11,12]$.

Despite Zambia having one of the world's highest estimated mortality rates from prostate cancer $[6,7]$ coupled with an increased suggested genetic predilection to the cancer [4], since the majority of the male population are black, no studies assessing knowledge, practice and attitude towards prostate cancer screening have been done. This study therefore sought to address the gap.

\section{Methods}

\subsection{Aims}

The aims of the study were to determine the knowledge, practice and attitude towards prostate cancer screening at Kitwe Teaching Hospital (KTH). In addition, the study also aimed to determine the association between demographics of participants and knowledge, knowledge of participants and attitude towards prostate cancer screening, knowledge of participants and prostate cancer screening practice as well as attitude of participants towards prostate cancer screening and prostate cancer screening practice.

\subsection{Study site and design}

The study was done at KTH in the Copperbelt province of Zambia. It is a tertiary referral hospital in the region whose catchment area includes Copperbelt, Luapula and North-western provinces. It has a bed capacity of 630 [13].

This was a descriptive cross-sectional study of knowledge, practice and attitude towards prostate cancer screening among male patients aged 40 years and above at $\mathrm{KTH}$. The study design was chosen because it is simple to use, cost-effective and time economic.

\subsection{Study participants}

The sample size was ascertained using the 'Stalcalc' function of Epi Info Version 7.1.5. In a month, nearly 419 male patients presented to the target areas for this study at $\mathrm{KTH}$, namely Out-Patient Department (OPD), medical and surgical admission wards. Since data for this study were collected in 1 month, 419 was used as the total population size. A confidence level was $95 \%$ and a confidence limit was 5\% (at 95\% confidence level) and the expected frequency was $50 \%$. Therefore, a sample size of 200 was calculated for this study. Study participants were randomly selected from target areas. All consenting male patients aged 40 years and above in the target areas for this study at KTH were enrolled until the targeted sample size was reached. Male patients aged less than 40 years and participants who did not give consent were excluded from the study.

\subsection{Study duration}

The study was done in a period of 6 months from April to September 2019. 


\subsection{Data collection and analysis}

The study objectives were explained to participants, and written and informed consent was obtained. Participants were enrolled utilizing a well-structured questionnaire as shown in "Appendix". The questionnaire collected demographic information including age, marital status, education and occupation. It also collected data on family history of cancer as well as knowledge, practice and attitudes towards prostate cancer screening. Translations to the questionnaire were done from English to a suitable local language according to the participant's preference. The responses were recorded as given by the participants.

Data collected during the study were checked for completeness and double-entered into the Epi Info version 7 software. Frequency tables and graphs were generated for relevant variables. The data were analysed using Statistical Package for Social Sciences (SPSS) version 23. For comparing associations between variables, Pearson Chisquare test was performed. A $p$ value of equal or less than 0.05 was considered significant. Binary logistic regression, as well as multivariate analysis, was done. Low knowledge was defined as scoring 1-3 correct responses in the knowledge section, moderate knowledge as scoring 4-6 correct responses and high knowledge as scoring 7-9. Positive attitude was defined as scoring 2 or more correct responses in the section assessing attitudes, while negative was defined as scoring less than 2 correct responses. Practice was assessed with a closed-ended question in the practice section.

Table 1 Demographics of study participants

\begin{tabular}{lrr}
\hline & Frequency & Percent \\
\hline Age & 149 & \\
$40-60$ years & 51 & 74.5 \\
$>60$ years & 200 & 25.5 \\
Total & & 100.0 \\
Religion & 200 & \\
Christian & & 100.0 \\
Marital status & 174 & \\
Married & 26 & 87.0 \\
Not married & 200 & 13.0 \\
Total & & 100.0 \\
Education level & 161 & \\
No formal education or primary education & 39 & 80.5 \\
Secondary or tertiary education & 200 & 19.5 \\
Total & & 100.0 \\
Occupation & 2 & 1.0 \\
Formal employment & 198 & 99.0 \\
Informal employment or unemployment & 200 & 100.0 \\
Total & & \\
\hline
\end{tabular}

\section{Results}

A total of 200 participants were enrolled.

\subsection{Background characteristics}

As illustrated in Table 1, more than half, 149 (74.5\%), of the participants in the study were in the $40-60$ years age range. All participants were Christians, 161 (80.5\%) had no formal education or had primary education, and 198 (99\%) were in informal employment or unemployed.

\subsection{Knowledge of prostate cancer}

Of the 200 participants enrolled, 67 (33.5\%) had heard about prostate cancer, while 133 (66.5\%) had never heard about it. Majority, 55.3\%, of the participants who had had heard about prostate cancer pointed to a doctor or nurse as a source of information as shown in Table 2.

Of the 200 participants enrolled in the study, 58 (29\%) expressed knowledge on prostate cancer. Among participants who had knowledge, majority of them, 37 (63.8\%) had low knowledge as shown in Fig. 1.

Participants who had secondary school or tertiary education were more knowledgeable about prostate cancer than those who did not have $(p<0.001)$. Participants who had heard about prostate cancer were more knowledgeable than those who had not $(p<0.001)$. Participants who had heard about prostate cancer had high levels of knowledge compared to those who had not $(p=0.009)$. Participants older than 60 years had more knowledge

Table 2 Sources of information on prostate cancer

\begin{tabular}{lcc}
\hline Source of information & Frequency & Percent \\
\hline TV & 10 & 14.9 \\
Radio & 17 & 25.4 \\
Nurse & 17 & 25.4 \\
Doctor & 20 & 29.9 \\
Read about it & 1 & 1.5 \\
Family and friends & 2 & 3.0 \\
Total & 67 & 100 \\
\hline
\end{tabular}

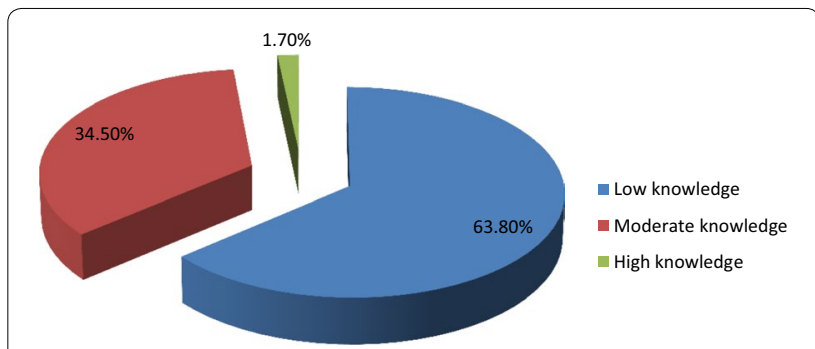

Fig. 1 Levels of knowledge 
on prostate cancer compared with those below 60 years $(p=0.026)$.

\subsection{Practice of prostate cancer screening}

Of the 200 participants, only 26 (13\%) had been screened in the last 2 years. Among participants who had screened, $20(76.9 \%)$ pointed out DRE as the method used, while 3 (11.5\%) pointed out PSA, 2 (7.69\%) reported both DRE and PSA, and 1 (3.85\%) did not know which screening method was used. Among the 26 participants that had screened in the last 2 years, $18(69.2 \%)$ had a positive prostate cancer outcome, while $8(30.8 \%)$ had a negative prostate cancer outcome. 199 (99.5\%) of the participants expressed intentions to screen in future.

Age above 60 years was associated with a positive prostate cancer outcome $(p=0.002)$. The study also found that participants who were knowledgeable about prostate cancer were more likely to undergo prostate cancer screening $(p<0.001)$ and that high level of knowledge was associated with prostate cancer screening practice $(p=0.024)$. Increasing age of participants (over 60) was also associated with prostate cancer screening practice in the last 2 years $(p<0.001)$.

\subsection{Attitude towards prostate cancer screening}

Among 200 participants enrolled in the study, 197 (98.5\%) had a positive attitude towards prostate cancer screening, while $3(1.5 \%)$ had a negative attitude. There were no statistically significant associations between age and attitude towards prostate cancer screening $(p=0.099)$, knowledge and attitude towards prostate cancer screening $(p=0.868)$ as well as between practice in the last 2 years and attitude towards prostate cancer screening $(p=0.291)$.

\subsection{Factors affecting prostate cancer screening practice} Binary logistic regression analysis was performed to identify factors that affect prostate cancer screening. As shown in Table 3, prostate cancer screening practice was

\begin{tabular}{|c|c|c|c|}
\hline Factors & Odds ratio & $\begin{array}{l}95 \% \text { Confidence } \\
\text { interval }\end{array}$ & $p$ value \\
\hline Age & 4.662 & $1.319-16.480$ & 0.017 \\
\hline Education & 3.773 & $1.058-13.451$ & 0.041 \\
\hline $\begin{array}{l}\text { Heard of prostate } \\
\text { cancer }\end{array}$ & 3.970 & $0.794-19.860$ & 0.093 \\
\hline Attitude & 0.468 & $0.011-19.504$ & 0.690 \\
\hline Knowledge & 6.114 & $1.276-29.286$ & 0.023 \\
\hline Family history of cancer & 25.673 & $3.107-212.109$ & 0.003 \\
\hline
\end{tabular}

associated with age $(p=0.017)$, education $(p=0.041)$, knowledge $(p=0.023)$ and family history of cancer $(p=0.003)$.

All factors that were significant in binary logistic analysis (with $p<0.05$ ) were analysed using multivariate logistic regression. A backward step-by-step elimination method was employed to manually eliminate factors with insignificant $p$ values. As illustrated in Table 4, only two factors remained statistically significant, namely knowledge and family history of cancer. Participants who had knowledge about prostate cancer were nearly 11 times more likely to practice prostate cancer screening than those who did not have $(p=0.001)$. Participants who had a family history of cancer were 26 times more likely to practice prostate cancer screening than those who had a negative family history of cancer $(p=0.002)$.

\section{Discussion}

The study targeted male patients aged 40 years and above due to available literature which indicates that prostate cancer screening should start at 40 years $[4,14]$. Literature indicates that the average age of a man to be diagnosed with prostate cancer is about 66 years and above $[4,15]$. Since the majority of the participants in the study, 149 (74.5\%), were in the 40-60 years age group, as also observed by Mofolo and colleagues in their study [16], there was an over representation of participants at the lowest risk of prostate cancer.

The study found low levels of awareness and knowledge. This is similar to findings of other studies done in other countries $[10,17]$. This implies that there is little sensitization being done to the public and expresses the need for more public sensitization campaigns utilizing both electronic and print media with the aim of early detection and treatment to improve the prognosis $[7,8$, 11]. However, other studies found high levels of awareness $[9,18-20]$. Of the studies that found high levels of knowledge, one of them was done on a group of public servants who were educated, had good access to health information and this was not a reflection of the general population who are mostly uneducated [9]. The study also demonstrated that majority of the participants who were knowledgeable about prostate cancer had low

\begin{tabular}{|c|c|c|c|}
\hline Factors & $\begin{array}{l}\text { Adjusted } \\
\text { odds } \\
\text { ratio }\end{array}$ & $\begin{array}{l}95 \% \text { Confidence } \\
\text { interval }\end{array}$ & $p$ value \\
\hline Knowledge & 10.974 & $2.634-45.728$ & 0.001 \\
\hline Family history of cancer & 26.106 & $3.258-209.193$ & 0.002 \\
\hline
\end{tabular}


level of knowledge which was consistent with findings by other studies $[10,18,19,21-23]$. This indicates a need for comprehensive knowledge on prostate cancer to promote early detection.

Participants with higher level of education were more knowledgeable about prostate cancer than those who had lower level of education or no formal education at all consistent with findings by similar studies done in other countries [16, 17, 21, 24]. However, some studies done in Nigeria and Kenya in 2018 did not find such an association $[10,19]$. In one of the studies that did not find an association between higher education and knowledge, the sample was drawn from a rural part of the country with more than $60 \%$ having no formal education or had primary education [19]. This could have resulted in the finding.

Participants older than 60 years had more knowledge on prostate cancer than those below 60 years as also demonstrated by Adibe et al. [24]. This highlights a possible bias that might be present in the provision of information on prostate cancer where individuals who are at an advanced age are educated about it because of their increased risk. It could also indicate the natural history of how older patients are more likely to have information about prostate cancer as they visit healthcare centres for urologic problems like benign prostate hyperplasia which are quiet frequent $[4,15]$.

In addition to health workers contributing to the increase in knowledge of prostate cancer, utilizing media platforms that are widely accessible such as radio presents a great opportunity to achieve this. A 2018 study by Kinyao and Kishoyian which assessed attitudes, perceived risk and intention in a rural county found that over $60 \%$ of the participants learnt about prostate cancer from the radio [19]. This shows how much more applicable this media platform can be in developing regions like Africa.

The low level of prostate cancer screening practice demonstrated in the study is consistent with findings from similar studies [10, 11, 19, 21, 25] though majority of participants in this study were willing to be screened after discussing about the condition with them consistent with a study done in Kenya [21]. However, it is inconclusive whether increased knowledge would increase screening as other factors apart from knowledge on prostate cancer appear to influence this practice. A similar study by Kinyao and Kishoyian in 2018 found that many participants had strong fatalistic attitudes towards screening such as "if I am meant to get prostate cancer, I will get it" and these appeared to influence screening [19]. Thus in sharing information on prostate cancer, cultural beliefs and fatalistic attitudes must also be addressed.
DRE was the most commonly used method of prostate cancer screening contrary to findings by similar studies done in Nigeria that found PSA to be the most commonly used method [24, 25]. This suggests a possible cost barrier to utilization of the PSA screening method in our sample.

The finding demonstrated in the study that participants were more likely to screen for prostate cancer if they were older than 60 years is consistent with findings of similar studies done in Uganda and Nigeria $[11,25]$. This implies that there is a risk of late presentation and consequently poor prognosis. As such, intensified public sensitization campaigns are needed to attain early detection and treatment as well as good prognosis. Participants who were more educated were more likely to undergo prostate cancer screening consistent with findings from a Nigerian study [25]. The statistically significant association between knowledge on prostate cancer and prostate cancer screening practice is consistent with other similar studies done $[10,11,21]$. This is another indication of the need to intensify prostate cancer sensitization campaigns.

The high positive attitude level demonstrated in the study was similar to findings from other studies done [9, 23, 24]. However, a Ugandan study found a negative attitude towards prostate cancer screening [11]. This could be because the study explored other factors under attitude that our study did not. The lack of any statistically significant association between age of participants and attitude towards prostate cancer screening concurs with findings from a study done in Uganda [11]. However, a 2017 study done in Nigeria found an association between age and attitude [24].

\section{Limitations of Study}

Generalizability of findings of this study must be done with caution since this was a hospital-based study. There is thus a need for more studies to be done in other institutions such as universities and colleges, urban and rural communities, district, general, central and other teaching hospitals to have comprehensive knowledge. In addition, certain aspects of knowledge were not assessed, for example, that prostate cancer can present without symptoms. As such, the study findings were limited to comparisons with studies that also did not assess the asymptomatic presentation of prostate cancer.

\section{Conclusion}

The study revealed low knowledge of prostate cancer, low prostate cancer screening practice and positive attitude of men towards prostate cancer screening. Practice of prostate cancer screening was associated with age, education level, knowledge and family history of cancer. 
Being the first study to assess knowledge, practice and attitude towards prostate cancer screening in Zambia, it has bridged the knowledge gap and has also provided valuable information for healthcare intervention.

\section{Supplementary information}

Supplementary information accompanies this paper at https://doi. org/10.1186/s12301-020-00067-0.

Additional file 1: Dataset for AFJU-D-19-00041R2 manuscript.

\section{Abbreviations}

DRE: Digital rectal examination; PSA: Prostate-specific antigen; OPD: OutPatient Department; TDRC: Tropical Diseases Research Centre; NHRA: National Health Research Authority; KTH: Kitwe Teaching Hospital; GLOBOCAN: Global Cancer Project.

\section{Acknowledgements}

First and foremost, I express my sincere, heartfelt and profound gratitude to the Almighty God for guiding, protecting and seeing me through the entire process of conducting this study. I am also grateful to my supervisor Prof. Victor Mwanasakale for his zeal and tireless efforts in seeing to it that this work becomes a reality. Let me also express my gratitude to Prof. Seter Siziya and the entire public health team for all the advice, encouragement. I would also like to thank the entire management at the Copperbelt University Michael Chilufya Sata School of Medicine for a friendly atmosphere. I would also like to thank my family back home for all the support and trust vested in me as well as my friends, roommates and class mates for the moral support.

A thesis submitted in partial fulfilment for the award of the Bachelors degree in medicine and surgery $(\mathrm{MBChB}$.

\section{Authors' contributions}

SG is the corresponding author, and KN and MV are the contributing authors. SG constructed the manuscript, collected data, analysed and interpreted data and also edited the manuscript. KN constructed the manuscript, analysed and interpreted data and extensively edited the manuscript. MV constructed the manuscript, extensively edited the manuscript and supervised this thesis study. All authors read and approved the final manuscript.

\section{Author's information}

The corresponding author (S.G) is currently pursuing his Bachelors degree in Medicine and Surgery (MBChB) at the Copperbelt University Michael Chilufya Sata School of Medicine in Zambia. K.N is a Pan African Organization for Health, Education and Research (POHER) scholar with a rich research background, who has presented at so many conferences. She was a recipient of the international research elective which took place at University of Missouri School of Medicine, USA, for 2-3 months. She holds a Bachelors Degree of Medicine and Surgery (MBChB) and graduated as the Best student. M.V was also the supervisor of this work. He holds BSc Human Biology, MBChB, MSC and a PhD in Parasitology and is an Associate Professor of Parasitology at the Copperbelt University Michael Chilufya Sata School of Medicine, Zambia.

\section{Funding}

This work was funded by the Government of the Republic of Zambia through the Ministry of Higher Education through its Higher Education Loans and Scholarships Board. As part of policy, the Ministry of Higher Education through its Higher Education Loans and Scholarships Board finances students in Higher Education institutions whose training demands the carrying out of research. Funding given covers for such expenses incurred during research such as printing and photocopying of data collection tools, transport charges for the researcher during the whole process and ethical clearance charges.

\section{Availability of data and material}

Data used in the study is available in additional file 1 captioned 'Dataset for AFJU-D-19-00041R2 manuscript' and authors agree to share it.
Ethics approval and consent to participate

A request to conduct the study was sought from the Tropical Diseases Research Centre (TDRC) research ethics committee (IRB Registration Number: 00002911, FWA Number: 00003729) as well as the National Health Research Authority (NHRA). Management at Kitwe Teaching Hospital was assured that confidentiality would not be breached and that the data obtained in the study would not be used for any other purpose besides that specified in the study protocol. Informed and written consent was obtained from participants. During data collection, no identifying images or other personal or clinical details of participants were collected. They were treated with at-most respect and dignity and their rights to privacy and confidentiality were not violated at any point.

\section{Consent for publication}

In this study, no data that could compromise the anonymity of participants such as images or other personal or clinical details were collected. As such, it was not applicable.

\section{Competing interest}

The authors declare that they have no competing interests.

\section{Author details}

${ }^{1}$ Michael Chilufya Sata School of Medicine, The Copperbelt University, P.O. Box 71191, Ndola, Zambia. ${ }^{2}$ Pan - African Organization for Health, Education and Research (POHER), Lusaka, Zambia.

\section{Appendix: Questionnaire}

Topic: assessment of knowledge, practice and attitude towards prostate cancer screening among male patients aged 40 years and above at Kitwe Teaching Hospital.

NAME OF INTERVIEWER

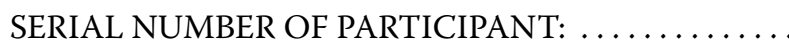
DATE:

\section{Section A: demographic characteristics}

Instruction: Please, tick as appropriate.

1. Age:... years

2. Marital status: Single [] Married [] Divorced [] Separated []

3. Religion: Christian [] Muslim [] Traditional []

4. Educational level: Primary [] Secondary [] Tertiary [] No formal education []

5. Occupation: Trader [] Civil servant [] Taxi driver [] Businessman [] Electrician [] Mechanic [] Barber [] Other (please specify)

\section{Section B: family history of cancer}

6. Does anyone in your family have cancer? Yes [] No [] If Yes

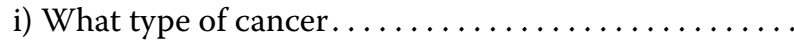

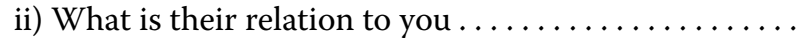
7. Has anyone in your family died of Cancer Yes [] No [] If Yes,

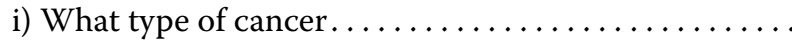

ii) What is their relation to you $\ldots \ldots \ldots \ldots \ldots \ldots \ldots$ 


\section{Section C: knowledge}

8. Have you heard of prostate cancer before: Yes [] No [] If Yes,

i) Where did you hear it from Friends [] Read about it [] TV [] Radio [] Doctor [] Nurse [] Relative []

ii) Which gender does prostate cancer affect Men only [] Women only [] Both men and women [] I do not know []

iii) Which of the following factors could make a person more likely to develop prostate cancer. Please tick as many as possible

a) Family history of the disease [] b) Drinking alcohol [] c) Age [] d)Exercise [] e) Diet [] f) Smoking []

9. Do you know symptoms of prostate cancer Yes [] No []

If Yes, what are they? Tick as many as possible. a) Excessive urination at night [] b)Headache [] c) blood in urine [] d) High temperature [] e) Bone pain [] f) Painful sex [] g) Loss of sex drive [] h) Infertility [] i) cough []

10. Is prostate cancer preventable Yes [] No [] I do not know []

If yes,

a) How can it be prevented? Genital hygiene [] regular screening [] condom use [] use of right diet [] avoiding many sexual partners []

11. Is prostate cancer curable Yes [] No [] I don't know []

\section{Section D: practice}

12. Have you been screened for prostate cancer within the last two years? Yes [] No []

If yes,

a) Which method was used Prostate Specific Antigen (PSA)

[] Digital Rectal Examination (DRE) [] I do not know []

b) What was the outcome of the screening? Positive [] Negative []

13. Do you have any intention of getting screened in the nearest future? Yes [] No []

\section{Section E: attitude towards prostate cancer screening}

14. Prostate cancer screening is good Yes [] No []

15. Going for prostate cancer screening is a waste of time Yes [] No []

16. Prostate cancer screening has side effects that can cause harmful effects to the body Yes [] No []

Thank You!

Received: 1 November 2019 Accepted: 16 September 2020

Published online: 07 November 2020
References

1. Blandy J, Kaisary A (2009) Lecture notes urology, 6th edn. Wiley, Hoboken

2. Shenoy KR, Shenoy A (2019) Manipal manual of surgery, 4th edn. CBS Publishers Pvt Ltd., Shenoy Nagar

3. Desousa A, Sonavane S, Mehta J (2012) Psychological aspects of prostate cancer. Prostate Cancer and Prostatic Dis 15(2):120-127

4. American Cancer Society (2019) Cancer facts and figures, 2019. American Cancer Society, Atlanta

5. GloboCan (2018). http://globocan.iarc.fr

6. GloboCan (2012). http://globocan.iarc.fr

7. National Cancer Control Strategic Plan 2016-2021. Ministry of Health Zambia

8. So WK, Choi KC, Tang WP, Lee PC, Shiu AT, Ho SS et al (2014) Uptake of prostate cancer screening and associated factors among Chinese men aged 50 or more: a population-based survey. Cancer Biol Med 11(1):56-63

9. Oranusi CK, Mbieri UT, Oranusi IO, Nwofor AME (2012) Prostate cancer awareness and screening among male public servants in Anambra State Nigeria. Afr J Urol 18(2):72-74

10. Awosan KJ, Yunusa EU, Agwu NP, Taofiq S (2018) Knowledge of prostate cancer and screening practices among men in Sokoto, Nigeria. Asian J Med Sci 9(6):51-56

11. Nakandi H, Kirabo M, Semugabo C, Kittengo A, Kitayimbwa P, Kalungi S, Maena J (2013) Knowledge, attitudes and practices of Ugandan men regarding prostate cancer. Afr J Urol 19(4):165-170

12. Ito K (2014) Prostate cancer in Asian men. Nat Rev Urol 11:197

13. Sichula M, Kabelenga E, Mwanakasale V (2018) Factors influencing malnutrition among under five children at Kitwe Teaching Hospital, Zambia. Int Curr Innov in Adv Res 1(7):9-18

14. Canadian National Institute of Health (2013)

15. Cancer Diseases Hospital (2013) Annual report

16. Mofolo N, Betshu O, Kenna O, Koroma S, Lebeko T, Claassen FM, Joubert Gina (2015) Knowledge of prostate cancer among males attending a Urology clinic, a South African study. SpringerPlus 4:67

17. Kabore FA, Kambou T, Zango B, Ouédraogo A (2014) Knowledge and awareness of prostate cancer among the general public in Burkina Faso. J Cancer Educ 29:69-73. https://doi.org/10.1007/s13187-013-0545-2

18. Muhammad FHMS, Soon LK, Azlina Y (2016) Knowledge, awareness and perception towards prostate cancer among male public staffs in Kelantan. Int J of Public Health Clin Sci 3(6):105-115

19. Kinyao M, Kishoyina G (2018) Attitude, perceived risk and intention to screen for prostate cancer by adult men in Kasikeu sub location, Makueni County, Kenya. Ann Med Health Sci Res 8(3):125-132

20. Agbugui JO, Obarisiagbon EO, Nwajei CO, Osaigbovo EO, Okolo JC, Akinyele AO (2013) Awareness and knowledge of prostate cancer among men in Benin City, Nigeria. J Med Biomed Res 12(2):42-47

21. Wanyagah P (2013) Prostate cancer awareness, knowledge, perception on self-vulnerability and uptake of screening among men in Nairobi. Kenyatta University, Kenya

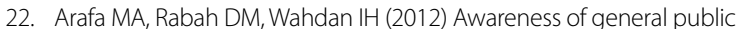
towards cancer prostate and screening practice in Arabic communities: a comparative multi-center study. Asian Pac J Cancer Prev 13:4321-4326

23. Makado E, Makado RK, Rusere MT (2015) An assessment of knowledge of and attitudes towards prostate cancer screening among men aged 40 to 60 years at Chitungwiza Central Hospital in Zimbabwe. Int J Humanit Soc Stud 3(4):45-55

24. Adibe MO, Oyine DA, Abdulmuminu I, Chibueze A (2017) Knowledge, attitudes and perceptions of prostate cancer among male staff of the University of Nigeria. Asian Pac J Cancer Prev 18(7):1961-1966

25. Ebuechi OM, Otumu IU (2011) Prostate screening practices among male staff of the University of Lagos, Lagos, Nigeria. Afr J Urol 17(4):122-134

\section{Publisher's Note}

Springer Nature remains neutral with regard to jurisdictional claims in published maps and institutional affiliations. 